\title{
EVOLUÇÃO DOS GASTOS PÚBLICOS ESTADUAIS EM CAPITAL HUMANO E EM INFRAESTRUTURA FÍSICA NOS ESTADOS BRASILEIROS ${ }^{1}$
}

\author{
Aline Cristina $\mathrm{Cruz}^{2}$ \\ Daniela Almeida Raposo ${ }^{3}$ \\ Erly Cardoso Teixeira ${ }^{4}$ \\ Robson Miranda Silva ${ }^{5}$ \\ Rogerio Cesar Corgosinho 6
}

\begin{abstract}
RESUMO: O objetivo principal deste trabalho é avaliar a evolução dos gastos públicos em capital humano e físico e a evolução de alguns dos principais indicadores socioeconômicos nos diversos estados brasileiros, de forma a extrair conclusões sobre a eficácia destes dispêndios do Governo, quanto à meta de aumento do bem estar das famílias dos diversos estados do país. As principais conclusões atestam eficácia dos dispêndios públicos em infraestrutura rodoviária e em educação e saúde quanto aos avanços na malha rodoviária e no estoque de capital humano, respectivamente, a partir da análise da estrutura socioeconômica dos estados brasileiros. Fica claro que os estados do Sul e Sudeste continuam a concentrar riqueza e maior infraestrutura em detrimento dos demais. Entretanto, houve melhora geral no país, nos anos de 2007 e 2008, em relação à década de 1980, a exemplo da diminuição da mortalidade e da melhora de indicadores de escolaridade média, PIB per capita, condição das rodovias federais e de concentração de renda, em todos os estados brasileiros.
\end{abstract}

Palavras- chave: gastos públicos, capital humano, infraestrutura física.

\section{EVOLUTION OF STATE PUBLIC SPENDING IN HUMAN CAPITAL AND PHYSICAL INFRASTRUCTURE IN THE BRAZILIAN}

\begin{abstract}
The main objective of this paper is to analyses the evolution of public expenditures in human and physical capital and some of the principals socioeconomic indicators in the Brazilian states. The goal is obtain conclusions about the efficacy of the Government to increasing the well being of families. The results indicate efficacy of the expenditures in road infrastructure and education and health related to improvement of roads and stock of human capital. It is evident the South and Southeast states keep concentrating better level of income and infrastructure. However, there was progress of general way in 2007 and 2008 relative to eighties. There was reduction of the tax mortality and advances in years of study, per capita income, federal roads conditions and the income concentration in all states.
\end{abstract}

Key-words: public expenditures, human capital, physical infrastructure.

\footnotetext{
${ }^{1}$ Agradecemos ao apoio financeiro da Fundação de Amparo à Pesquisa do Estado de Minas Gerais (Fapemig).

${ }^{2}$ Professora Adjunta II do Departamento de Ciências Econômicas da Universidade Federal de São João del Rei (UFSJ). E-mail: alinecruz@ufsj.edu.br.

${ }^{3}$ Professora Adjunta II do Departamento de Ciências Econômicas da Universidade Federal de São João del Rei (UFSJ). E-mail: daniraposto@ufsj.edu.br.

${ }^{4}$ Professor Titular do Departamento de Economia Rural da Universidade Federal de Viçosa (UFV). E-mail: teixeira@ufv.br.

${ }^{5}$ Graduando em Ciências Econômicas da Universidade Federal de São João del Rei. E-mail: robsonmagic@yahoo.com.br.

${ }^{6}$ Graduando em Ciências Econômicas da Universidade Federal de São João del Rei. E-mail: rogeriocorgosinho@ hotmail.com.
}

Recebido em 05/11/2013

Aceito em 22/11/2013 


\section{I - INTRODUÇÃO}

O questionamento controverso sobre o tamanho e o papel do Estado é foco de vários estudos, os quais, por vezes, buscam levantar justificativas para as formas de intervenção estatal no exercício de suas funções distributiva, alocativa e de estabilização. Em meio a esta discussão, torna-se importante avaliar em que medida as despesas públicas contribuem para o crescimento econômico e para a redução da pobreza em países com características tão específicas, como o Brasil, dada sua condição de país continental. De fato, dadas as disparidades entre suas diversas regiões, torna-se evidente o desafio de todas as esferas do Governo de conseguir promover efeitos em indicadores sociais e econômicos em todos os estados do país, com reflexos positivos na qualidade de vida da parcela da população com menor poder aquisitivo.

O que se observa é que os debates ligados ao papel do Governo são marcados, também, por questões políticas e pela exigência de que os gastos públicos devem ser acompanhados de ajustes fiscais e metas de superávit primário. Há ainda o receio do crescimento da dívida pública e do retorno da instabilidade monetária vivenciada nas décadas de 1980 e de 1990, de modo, que, desde então, persistem as metas de controle inflacionário e de equilíbrio externo lado a lado com cenários econômicos marcados por modestas taxas de crescimento.

A despeito da eficácia das instituições públicas na promoção do crescimento econômico, muitos estudos têm reconhecido que os gastos públicos em capital humano e infraestrutura física são instrumentos chave no processo de desenvolvimento econômico. A importância do direcionamento das políticas públicas encontra respaldo em estudos como de Loayza et al. (2004), cujas projeções de crescimento para o período 2000-10 mostraram que o Brasil apresentaria crescimento do PIB per capita relativamente superior às demais nações latino-americanas. Mais especificamente, a taxa de crescimento seria 2,5\% superior à taxa da década de noventa $(1,07 \%)$, se realizados aprimoramentos nas políticas estruturais, ou seja, fortes expansões em educação e infraestrutura pública. Os números mostraram que investimentos em educação e em infraestrutura contribuiriam com aproximadamente um quarto do aumento na taxa de crescimento do PIB per capita brasileiro.

Tais resultados vão de encontro ao trabalho de Cruz et al. (2010), cujas conclusões mostram que as despesas em educação e saúde, bem como em estradas e energia são eficientes quanto à melhora da qualidade do capital nacional humano e físico. Especificamente, fica comprovado que maior nível de escolaridade, melhores condições de 
Evolução dos gastos públicos estaduais em capital humano e em infraestrutura física nos Estados brasileiros.

saúde, acréscimos na formação bruta de capital fixo, além de melhorias na infraestrutura rodoviária e energética, contribuem significativamente para elevar a renda per capita da população brasileira e a produtividade total dos fatores. O trabalho mostra, sobretudo, que as despesas públicas estaduais e federais em infraestrutura e capital humano geraram crescimento econômico acompanhado de queda na pobreza, no Brasil, no período de 1980 a 2007.

Ainda sob esta perspectiva, Ferranti et al. (2004) defende que a eficiência dos gastos públicos e progressos nas áreas de educação, governança, infraestrutura e estabilidade macroeconômica estão associados ao crescimento econômico e à queda na desigualdade de renda. Tais políticas são consideradas como "win-win", pois reduzem a pobreza e permitem o crescimento da economia.

Nesse sentido, dada a importância da atuação eficaz do Governo como forma de promover o crescimento econômico que beneficie também os pobres (crescimento pró-pobre), torna-se importante avaliar a evolução das despesas públicas voltadas ao aprimoramento do estoque de capital físico e humano nas diversas regiões do país. Embora o Brasil seja uma nação desigual exposta ao desafio histórico de combate à exclusão social, é pertinente levantar evidências de que as esferas governamentais tem seguido uma trajetória de expansão de seus gastos em capital social em todas as regiões do Brasil acompanhada de eficácia no cumprimento de suas metas primeiras.

No que concerne à atuação do Estado, sabe-se da importância de políticas de gasto social, a exemplo do Programa Bolsa Família, carro chefe da política econômica brasileira atual. Todavia, deve-se dar prioridade às políticas públicas que permitam retirar de maneira definitiva a parcela da população de menor poder aquisitivo da condição de dependente de apoio financeiro estatal. Neste sentido, o objetivo principal deste trabalho é avaliar a evolução dos gastos públicos em capital humano e físico, bem como a evolução de alguns dos principais indicadores socioeconômicos nos diversos estados brasileiros, de forma a extrair conclusões sobre a eficácia destes dispêndios do Governo, quanto à meta de aumento do bem estar das famílias dos diversos estados do país. Diante dos objetivos propostos, a metodologia usada tem como norte a Estatística Descritiva, por meio de análise tabular e gráfica. Para tal, no que diz respeito à estrutura do trabalho, além da introdução e das conclusões finais, este estudo apresenta uma seção expondo uma breve revisão de literatura seguida da seção de análise e discussão dos resultados. 


\section{2- REVISÃO DE LITERATURA}

O século XXI vem revelando-se como o tempo das inovações, da valorização do conhecimento e tais transformações tecnológicas provocam aumento da demanda por trabalhadores qualificados. Como resultado, os rendimentos da massa de trabalhadores mais qualificados crescem, aumentando o hiato de salários entre os mesmos e a mão de obra não qualificada, o que tende a contribuir para o aumento da desigualdade social. É importante atentar para o fato de que o termo desigualdade social envolve desigualdade educacional, a desigualdade de oportunidades, e a desigualdade de renda, entre outros aspectos. Em síntese, a desigualdade econômica percebida entre os indivíduos de uma nação denomina-se de desigualdade social, levando-se em consideração a distribuição da renda. A razão é que o aumento de renda não proporciona a inserção social, diretamente, uma vez que depende de variáveis multidimensionais. Desta forma, políticas que visam apenas ao aumento da renda média para a redução da desigualdade são apenas políticas de curto prazo, com efeitos limitados sobre outras áreas.

$\mathrm{Na}$ discussão do conceito de desigualdade social, devem ser inclusos o acesso aos serviços de educação, saúde, inclusão digital, a participação política, entre outros correlatos. Assim, o simples aumento de renda, embora seja condição necessária, não é condição suficiente para a obtenção desses serviços. Nessa ótica, Sen (1983) introduz a ideia da oportunidade de escolha. Para o autor, não é condição suficiente aumentar os recursos financeiros do indivíduo, mas é, sumariamente, importante ampliar suas oportunidades de escolhas, no intuito de elevar o nível de seu bem-estar. Tal feito é possível por meio do investimento em capital humano, o qual poderá gerar agregação de conhecimento e, com isso, elevar a renda, permitir o acesso à saúde e a maior participação nas decisões políticas do país, ampliando, por sua vez, as oportunidades do indivíduo de atuar enquanto cidadão.

Nesse contexto, insere-se a importância da discussão da eficácia do Governo de promover o crescimento pró-pobre. Em estudos como os de White e Anderson (2000), de Manso e Barreto (2009) e de Kalkwani e Pernia (2000), a discussão do termo pró-pobre trata de dois indicadores: desigualdade de renda e crescimento da renda média. Para Lopez (2004a), o crescimento "pró-pobre" se dá quando a participação da renda dos mais pobres da população aumenta mais que proporcionalmente ao crescimento da renda média da economia - queda da desigualdade lado a lado com o crescimento dos rendimentos. Já White e Anderson (2000) identificam o crescimento pró-pobre, se há aumento da renda per capita dos mais pobres e, por conseguinte, queda da desigualdade absoluta. No entanto, para os estudiosos 
Evolução dos gastos públicos estaduais em capital humano e em infraestrutura física nos Estados brasileiros.

defensores do crescimento da renda, vale mencionar Ravallion e Chen (2003), que argumentam que o crescimento pró-pobre está relacionado à capacidade de reduzir a pobreza para determinada medida pré-estabelecida, independente do ocorrido com o nível de desigualdade.

Do ponto de vista da definição da causa principal da pobreza no Brasil, a verdade é que a busca de soluções para a pobreza exige um esforço mais amplo inserido na busca constante de desenvolvimento social e econômico. Para isso, as ações governamentais são de suma importância, de modo que é essencial avaliar a eficácia e a eficiência do gasto público na consecução do objetivo inicial. No que concerne às políticas de crescimento, os mais pobres podem ser beneficiados por intermédio do aumento da demanda por trabalho e consequente aumento salarial.

No entanto, o processo de crescimento está cada vez mais centrado em tecnologia, inovação, e na agregação de conhecimento. Desta forma, maiores níveis de crescimento melhoram a situação daqueles que possuem nível educacional maior. Dada a capacidade de acesso à educação, pode-se perceber que os mais pobres têm mais dificuldade de obter o mesmo nível educacional em relação aos mais ricos (CASTRO, 2009). Assim, se o processo de crescimento econômico aumenta mais a renda dos trabalhadores com maior nível educacional, os quais, neste caso, representam os indivíduos que já se encontram em famílias com maior renda, pode-se crer que o crescimento econômico tende a perpetuar a desigualdade. Tal resultado reforça a visão de que as políticas de combate à pobreza por meio do crescimento são mais eficazes, quando acompanhadas do processo de redistribuição de renda. Como documentado em Lopez (2004b), alguns modelos mostram que a desigualdade pode tanto prejudicar o crescimento econômico, assim como também estimulá-lo.

Analisando-se os gastos públicos que alteram a relação entre os fatores de produção, e deste modo, a dinâmica do mercado de trabalho, pode-se destacar os gastos em capital humano e capital físico. O gasto em capital humano é realizado via gastos em educação, saúde, melhores condições de vida, enquanto o gasto em capital físico implica em dispêndios em infraestrutura (transporte, energia, telecomunicações), sendo que ambos influenciam a produção, ao alterar a produtividade total dos fatores.

O gasto em capital humano melhora o nível de produtividade do trabalhador, aumentando sua produção por hora e, consequentemente, sua remuneração. Quanto maior o investimento em capital humano realizado pelo indivíduo, maior a sua probabilidade de inserção no mercado de trabalho e de possuir rendimentos que lhes assegure um padrão de vida aceitável. 
Outro efeito advindo do gasto em capital humano é sua relação com o crescimento econômico. Para Ferreira et al. (2003), supondo rendimentos de capital físico e trabalho decrescentes, uma explicação para o crescimento de longo prazo seria o avanço tecnológico, dado que este eleva a produtividade dos fatores de produção. Segundo Blackburn et al. (2000), a acumulação de capital humano é fator determinante na determinação do progresso tecnológico. Em relação aos países subdesenvolvidos, Keller (2003) ressalta que, estando os investimentos tecnológicos centrados em poucos países altamente desenvolvidos, a difusão de tecnologia torna-se um importante determinante da distribuição da renda mundial. Em relação à assimilação de novas tecnologias, Foster e Rosenzweig (1996) argumentam que pessoas mais educadas são mais capazes de assimilar novas informações e de gerir novas tecnologias, tirando maiores proveitos de mudanças tecnológicas.

Sob esta perspectiva, percebe-se a importância de investimentos em capital humano. Considerando-se, ainda, as imperfeições dos mercados, as quais alteram as decisões dos gastos individuais dos agentes, são essenciais os investimentos públicos em capital humano como forma de complementar os gastos individuais da parcela da população afetada pelas imperfeições dos mercados. Ademais, o gasto em capital humano tem a particularidade de permitir que o indivíduo consiga reduzir seu nível de pobreza, por meio do próprio esforço, sendo assim uma política sustentável de combate à pobreza e de promoção do crescimento econômico, via aumento da produtividade.

Já o gasto público em capital físico possui alto nível de dinamismo econômico. Segundo Ferreira (1996) e Ferreira e Malliagros (1998), há forte relação entre investimento em infraestrutura (energia, telecomunicações e transportes) e produto, dada a variação da elasticidade-renda de longo prazo desses investimentos entre 0,55 e 0,70. Para diversos autores (RAM, 1986; BARRO, 1990 e ASCAHUER, 1989), o gasto público em capital físico altera a produtividade do setor privado, aumentando o crescimento econômico. Em relação às economias mais atrasadas, alguns autores (VANDENBUSSCHE ET AL., 2006; ROCHA, 2011) argumentam que essas economias demandam mais recursos estruturais do que investimentos em tecnologia, ciência e inovação. Para Hirschman (1961), devido à elevada razão capital/produto necessário para fornecer serviços de infraestrutura e devido ao elevado volume demandado por esses investimentos, estes devem ser mantidos pela administração publica ou que esta regule os agentes que porventura forneçam esses serviços. 
Evolução dos gastos públicos estaduais em capital humano e em infraestrutura física nos Estados brasileiros.

\section{3 - ANÁLISE E DISCUSSÃO DOS RESULTADOS}

Esta seção tem por objetivo apresentar a análise da evolução das principais variáveis socioeconômicas referentes aos estados brasileiros, com a proposta de descrever as diferenças relativas dos indicadores econômicos destas unidades federativas. Os aspectos abordados dizem respeito à desigualdade de renda, estoque de capital físico e humano, categorias de gastos públicos, renda per capita e pobreza nos estados brasileiros, no período de 1985 a 2008. Ao comparar tais dados, nota-se considerável heterogeneidade socioeconômica entre os estados membros. A seguir, na Tabela 1, é possível observar, de maneira comparativa, o nível de desigualdade de renda dos estados brasileiros, por meio da análise do índice de Gini, no período de 1985 a 2008; e também verificar a evolução do índice, por meio do valor máximo e mínimo de cada estado.

Tabela 1 - Índice de Gini, estados brasileiros, 1985-2008

\begin{tabular}{l|c|c|c}
\hline \multicolumn{1}{c|}{ Estados } & Média & Máximo & Mínimo \\
\hline Acre & 0,58 & 0,63 & 0,50 \\
\hline Alagoas & 0,59 & 0,64 & 0,55 \\
\hline Amazonas & 0,54 & 0,58 & 0,50 \\
\hline Amapá & 0,51 & 0,65 & 0,42 \\
\hline Bahia & 0,59 & 0,64 & 0,55 \\
\hline Ceará & 0,60 & 0,66 & 0,53 \\
\hline Distrito Federal & 0,61 & 0,63 & 0,58 \\
\hline Espírito Santo & 0,58 & 0,65 & 0,52 \\
\hline Goiás & 0,56 & 0,63 & 0,51 \\
\hline Maranhão & 0,56 & 0,61 & 0,50 \\
\hline Minas Gerais & 0,56 & 0,61 & 0,51 \\
\hline Mato Grosso do Sul & 0,56 & 0,63 & 0,52 \\
\hline Mato Grosso & 0,56 & 0,62 & 0,50 \\
\hline Pará & 0,55 & 0,61 & 0,49 \\
\hline Paraíba & 0,60 & 0,65 & 0,56 \\
\hline Pernambuco & 0,59 & 0,63 & 0,55 \\
\hline Piaú́ & 0,61 & 0,66 & 0,55 \\
\hline Paraná & 0,55 & 0,60 & 0,49 \\
\hline Rio de Janeiro & 0,56 & 0,65 & 0,54 \\
\hline Rio Grande do Norte & 0,58 & 0,62 & 0,55 \\
\hline Rondônia & 0,53 & 0,58 & 0,45 \\
\hline Roraima & 0,51 & 0,58 & 0,42 \\
\hline Rio Grande do Sul & 0,54 & 0,59 & 0,50 \\
\hline Santa Catarina & 0,50 & 0,56 & 0,46 \\
\hline Sergipe & 0,58 & 0,62 & 0,53 \\
\hline São Paulo & 0,53 & 0,55 & 0,48 \\
\hline Tocantins & 0,57 & 0,63 & 0,52 \\
\hline BRASIL & 0,59 & 0,63 & \\
\hline Fon & 0,54 & \\
\hline
\end{tabular}

Fonte: Elaborada pelos autores com base em dados do Ipeadata (2012). 
Primeiramente, observa-se que os estados da região Nordeste, em sua maioria, detêm os maiores índices de desigualdade de renda, sendo que a maioria tem índice de Gini maior que a média nacional $(0,59)$. Isso é visto, sobretudo, nos estados do Piauí e Paraíba, com índices de 0,610 e 0,609, respectivamente. Com perfil distinto, tem-se a região Sul, a exemplo do estado de Santa Catarina, com índice de 0,508. No Sudeste, há relativa homogeneidade, em termos de concentração de renda entre seus estados membros, haja vista o índice para o Rio de Janeiro de 0,569 e de Minas Gerais, na casa de 0,566. O estado paulista, por sua vez, aponta índice de 0,532, ou seja, menor que a média nacional.

A análise da concentração de renda nos estados de Roraima, Amapá e Distrito Federal assume destaque. Os dois primeiros sobressaem devido à relativa baixa desigualdade da renda, com índices de Gini de 0,513 e 0,512, respectivamente. Entre as justificativas para este cenário está o fato de grande parte da população de Roraima e Amapá ser constituída por indivíduos pobres. São, respectivamente, 118.337 e 206.168 pessoas com renda inferior à linha de pobreza, sendo que, em 2010, a população nestes estados era de 450.479 e 669.526 habitantes, respectivamente. Isso indica que $26 \%$ da população de Roraima são indivíduos pobres e, no caso de Amapá, este percentual é 30,8\%, revelando valores extremamente elevados. Desse modo, fica claro que há baixa desigualdade de renda, nestes estados e paralelamente à alta incidência de pobreza. Já o Distrito Federal merece notabilidade por alcançar o maior índice de Gini médio $(0,610)$. A explicação pode estar relacionada aos evidentes altos salários dos funcionários dos poderes executivo e legislativo diferentemente do perfil restante da população que vive na capital nacional. Na Tabela 2, percebe-se que o maior valor médio de PIB per capita pertence ao Distrito Federal, fato, em parte, explicado pela concentração de funcionários públicos de elevada remuneração, neste estado. 
Evolução dos gastos públicos estaduais em capital humano e em infraestrutura física nos Estados brasileiros.

Tabela 2 - PIB per capita dos estados brasileiros, (em reais de 2000), 1985-2008

\begin{tabular}{l|c|c|c}
\hline \multicolumn{1}{c|}{ Estado } & Média & Máximo & Mínimo \\
\hline Acre & $3.470,00$ & $5.120,00$ & $2.900,00$ \\
\hline Alagoas & $2.740,00$ & $3.350,00$ & $2.280,00$ \\
\hline Amazonas & $7.180,00$ & $9.240,00$ & $5.910,00$ \\
\hline Amapá & $4.760,00$ & $6.610,00$ & $3.760,00$ \\
\hline Bahia & $3.760,00$ & $4.460,00$ & $3.240,00$ \\
\hline Ceará & $2.850,00$ & $3.680,00$ & $2.340,00$ \\
\hline Distrito Federal & $14.360,00$ & $23.800,00$ & $8.320,00$ \\
\hline Espírito Santo & $7.000,00$ & $10.470,00$ & $5.660,00$ \\
\hline Goiás & $4.600,00$ & $6.670,00$ & $3.460,00$ \\
\hline Maranhão & $1.860,00$ & $3.160,00$ & $1.400,00$ \\
\hline Minas Gerais & $6.000,00$ & $7.370,00$ & $5.420,00$ \\
\hline Mato Grosso do Sul & $5.760,00$ & $7.340,00$ & $4.730,00$ \\
\hline Mato Grosso & $5.560,00$ & $9.280,00$ & $3.720,00$ \\
\hline Pará & $3.520,00$ & $4.430,00$ & $2.880,00$ \\
\hline Paraíba & $2.600,00$ & $3.550,00$ & $2.020,00$ \\
\hline Pernambuco & $3.650,00$ & $4.200,00$ & $3.370,00$ \\
\hline Piauí & $1.870,00$ & $2.780,00$ & $1.400,00$ \\
\hline Paraná & $7.150,00$ & $8.990,00$ & $6.170,00$ \\
\hline Rio de Janeiro & $9.030,00$ & $11.200,00$ & $7.790,00$ \\
\hline Rio Grande do Norte & $3.220,00$ & $4.350,00$ & $2.660,00$ \\
\hline Rondônia & $4.420,00$ & $6.200,00$ & $3.050,00$ \\
\hline Roraima & $4.100,00$ & $6.130,00$ & $2.480,00$ \\
\hline Rio Grande do Sul & $8.560,00$ & $9.550,00$ & $7.760,00$ \\
\hline Santa Catarina & $8.060,00$ & $10.540,00$ & $6.820,00$ \\
\hline Sergipe & $4.100,00$ & $5.900,00$ & $3.250,00$ \\
\hline São Paulo & $10.900,00$ & $12.970,00$ & $9.680,00$ \\
\hline Tocantins* & $2.790,00$ & $5.290,00$ & $1.580,00$ \\
\hline BRASIL & $5.330,00$ & $7.280,00$ & $4.220,00$ \\
\hline Fon: Ea & \\
\hline
\end{tabular}

Fonte: Elaboração própria com base nos dados do Ipeadata/2012.

Nota: *Para o estado do Tocantins não há dados referentes ao período 1985-1988

Outra justificativa é que a concentração deste tipo de mão de obra aumenta o custo de vida, pois há muitas pessoas com salários altos, numa pequena área geográfica. Assim, o elevado custo de vida impede o movimento migratório para o Distrito Federal de pessoas com baixa renda. O segundo estado com maior PIB per capita é São Paulo, maior centro financeiro e industrial do país. Neste estado, a mobilidade social é maior, o que atrai pessoas menos qualificadas e oriundas de regiões mais pobres. O próprio movimento migratório para o estado paulista pode explicar, em parte, a razão pela qual o centro político do Brasil apresenta renda per capita maior que o centro financeiro e industrial. Observa-se ainda que, no Distrito Federal, durante o período, há tendência de crescimento do PIB per capita, com valor mínimo de $R \$ 8.320,00$, em 1985, e máximo bastante elevado e igual a $R \$ 23.280,00$, em 2008. Neste 
Revista Economia e Desenvolvimento, v. 25, n. 2, 2013.

último ano, o valor da renda per capita desse estado era quase duas vezes o valor da renda per capita paulista. O Rio de Janeiro, por sua vez, é o terceiro estado com maior valor médio de PIB per capita.

As unidades federativas de Maranhão, Piauí, Alagoas, Ceará e Tocantins apresentaram baixos valores de PIB per capita, demonstrando, uma vez mais, o fraco crescimento econômico das regiões Norte e Nordeste. Nestas, o estado que apresenta renda per capita mais elevada é o Amazonas. Outro fato a ser ressaltado é que $80 \%$ dos estados brasileiros apresentam valor máximo do PIB per capita, entre 2007 e 2008, fato que corrobora a tendência de crescimento econômico brasileiro recente. A seguir, a Tabela 3 revela informações sobre a escolaridade média da população.

Tabela 3 - Escolaridade média (anos de estudo), estados brasileiros, 1985 - 2007

\begin{tabular}{|c|c|c|c|}
\hline Estados & Média & Máximo & Mínimo \\
\hline Acre & 5,53 & 6,26 & 4,54 \\
\hline Alagoas & 3,67 & 4,80 & 2,05 \\
\hline Amazonas & 6,13 & 7,20 & 5,03 \\
\hline Amapá & 6,27 & 7,60 & 5,14 \\
\hline Bahia & 3,95 & 5,37 & 2,83 \\
\hline Ceará & 3,85 & 5,53 & 2,52 \\
\hline Distrito Federal & 7,98 & 9,40 & 6,63 \\
\hline Espírito Santo & 5,43 & 6,96 & 4,36 \\
\hline Goiás & 5,19 & 6,71 & 3,83 \\
\hline Maranhão & 3,44 & 5,22 & 2,14 \\
\hline Minas Gerais & 5,18 & 6,64 & 4,03 \\
\hline Mato Grosso do Sul & 5,37 & 6,73 & 4,15 \\
\hline Mato Grosso & 5,07 & 6,26 & 3,74 \\
\hline Pará & 5,38 & 6,08 & 4,82 \\
\hline Paraíba & 4,12 & 5,19 & 2,90 \\
\hline Pernambuco & 4,48 & 5,77 & 3,26 \\
\hline Piauí & 3,42 & 4,98 & 2,07 \\
\hline Paraná & 5,44 & 7,15 & 3,91 \\
\hline Rio de Janeiro & 6,86 & 8,13 & 5,86 \\
\hline Rio Grande do Norte & 4,41 & 5,78 & 3,22 \\
\hline Rondônia & 5,54 & 6,24 & 5,05 \\
\hline Roraima & 6,30 & 7,90 & 5,29 \\
\hline Rio Grande do Sul & 5,96 & 7,11 & 4,76 \\
\hline Santa Catarina & 5,81 & 7,33 & 4,47 \\
\hline Sergipe & 4,39 & 6,08 & 2,66 \\
\hline São Paulo & 6,43 & 7,94 & 5,12 \\
\hline Tocantins & 4,57 & 6,09 & 3,13 \\
\hline Brasil & 5,5 & 6,09 & 4,3 \\
\hline
\end{tabular}

Fonte: Elaborada pelos autores com base em dados do Ipeadata/2012.

Primeiramente, nota-se grande disparidade entre os estados quanto ao grau de instrução. A região Nordeste apresenta os menores valores, a exemplo do Piauí, com escolaridade média de 3,4 anos de estudo, por sua vez, o menor grau de escolaridade médio 
Evolução dos gastos públicos estaduais em capital humano e em infraestrutura física nos Estados brasileiros.

do Brasil. Ademais, sendo a escolaridade média nacional de 5,5 anos de estudo, vê-se que nenhum estado nordestino ultrapassou a média do país.

Apesar de alguns avanços, sabe-se que o Brasil tem condições de alcançar níveis de escolaridade média melhores. Na análise dos indicadores de educação, o baixo índice da região Nordeste contribui para que a média nacional seja reduzida. Estes números revelam que estas regiões são deficitárias quanto à qualidade da educação, apontando, desse modo, a necessidade de priorizar as políticas públicas que visam o desenvolvimento do capital humano localizados dessas áreas. Se por um lado, estados como Alagoas, Bahia, Ceará, Maranhão têm média inferior a quatro anos estudados, por outro lado, o Distrito Federal apresenta média de 7,9 anos de estudo, a maior do país. Destacam-se também a escolaridade média da população do Rio de Janeiro, definida em 6,86 anos de estudo, assim como a de São Paulo (6,4 anos), Amapá (6,2 anos), e Roraima (6,3 anos), sendo todas superiores à média nacional de 5,5 anos de estudo.

Outra observação importante é o fato de que os maiores valores para anos de estudo dizem respeito aos anos de 2006 e 2007, exceto Rondônia e Roraima, cuja escolaridade média máxima refere-se a 1999. Por outro lado, os valores mínimos são os de 1985 a 1989, excepcionalmente, para Roraima (2001) e Tocantins (1992), estado que nesses anos ainda fazia parte de Goiás. Entre as justificativas para tal fato estaria o momento político/econômico distinto ligado aos anos de 1980, cujas características eram, sobremaneira, divergentes da realidade do país a partir dos anos 2000.

Na Figura 1, pode-se visualizar o desvio padrão do nível médio de escolaridade. Observa-se que Sergipe, Paraná, Santa Catarina, Mato Grosso e Ceará foram os estados cujas variações da média de anos estudados são maiores, ao longo do período analisado. Rondônia apresenta desvio padrão de 0,365, ou seja, o menor do Brasil. Muito próximo desse estado estão Pará e Acre, com valores de 0,423 e 0,583, respectivamente. Conforme se observa, os estados da região Nordeste obtiveram desvio padrão altos, se comparados com o restante do país. 


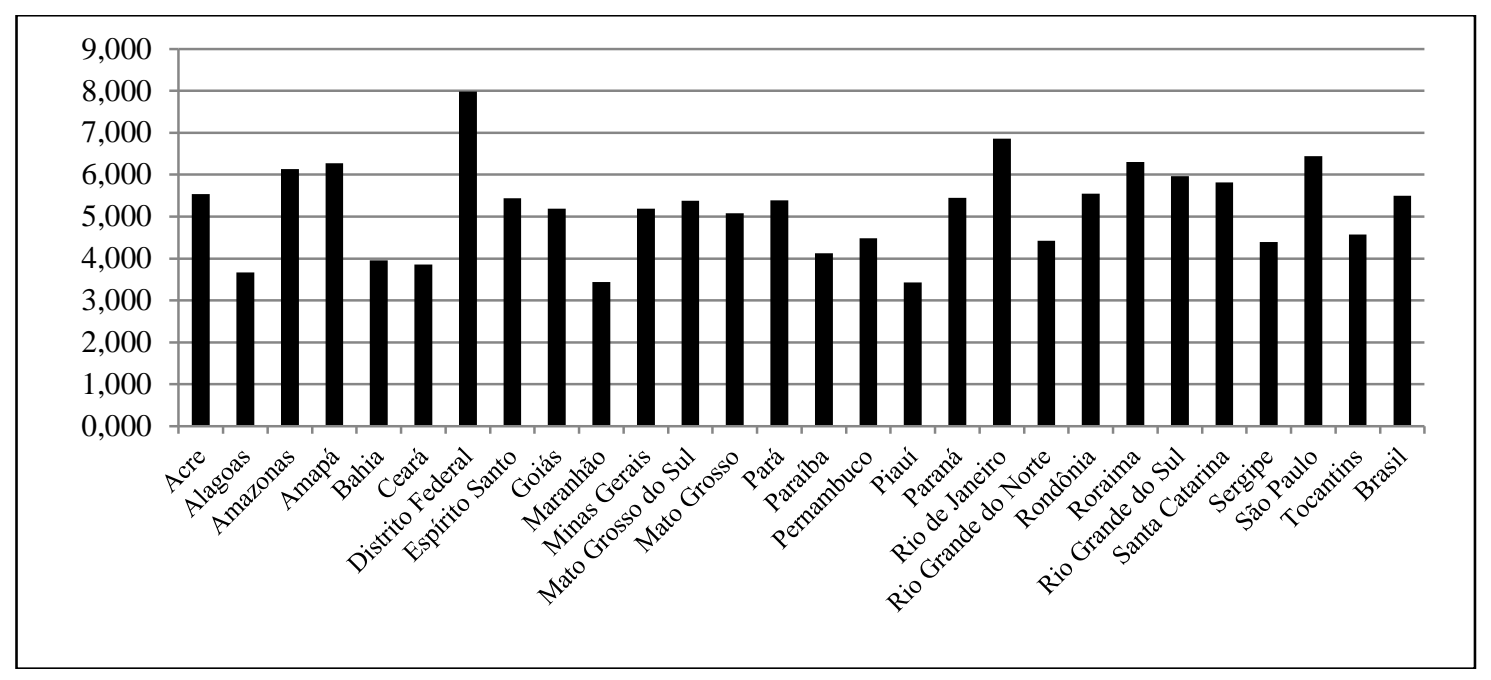

Figura 1 - Desvio padrão das escolaridades médias, estados do Brasil

Fonte: Elaborada pelos autores com dados do Ipeadata/2012.

Diante da proposta de avaliar a eficácia dos gastos públicos estaduais para elevar a escolaridade média, é importante apresentar informações sobre os dispêndios do Governo em educação e cultura (Tabela 4). Assume destaque nesta tabela o estado de Roraima, o qual obteve o maior gasto per capita (R\$268,87) nessa área. Em seguida está o Amapá, com média anual de $\mathrm{R} \$ 257,11$ de despesa per capita nesta função. Os dispêndios desses dois estados em educação/cultura corroboram seus devidos resultados, no que diz respeito aos anos estudados, posto que estão entre os melhores do país (Tabela 3). 
Evolução dos gastos públicos estaduais em capital humano e em infraestrutura física nos Estados brasileiros.

Tabela 4 - Gasto médio per capita em educação e cultura dos estados brasileiros, 1985 a 2007

\begin{tabular}{l|c|c|c}
\hline \multicolumn{1}{c|}{ Estados } & Média & Máximo & Mínimo \\
\hline Acre & 218,66 & 724,03 & 0,0083 \\
\hline Alagoas & 60,86 & 164,97 & 0,0044 \\
\hline Amazonas & 110,27 & 324,80 & 0,0063 \\
\hline Amapá & 257,11 & 715.38 & 0,0031 \\
\hline Bahia & 65,73 & 166,29 & 0,0012 \\
\hline Ceará & 76,27 & 239,97 & 0,0035 \\
\hline Distrito Federal & 255,92 & 71,92 & 0,0020 \\
\hline Espírito Santo & 87,68 & 271,95 & 0,0056 \\
\hline Goiás & 91,28 & 276,58 & 0,0052 \\
\hline Maranhão & 55,44 & 165,36 & 0,0016 \\
\hline Minas Gerais & 95,39 & 211,04 & 0,0052 \\
\hline Mato Grosso do s Sul & 105,02 & 299,81 & 0,0073 \\
\hline Mato Grosso & 103,17 & 310,56 & 0,0071 \\
\hline Pará & 59,40 & 160,56 & 0,0031 \\
\hline Paraíba & 73,90 & 193,19 & 0,0027 \\
\hline Pernambuco & 46,17 & 153,72 & 0,0035 \\
\hline Piauí & 69,53 & 195,21 & 0,0049 \\
\hline Paraná & 109,46 & 386,02 & 0,0057 \\
\hline Rio de Janeiro & 126,37 & 361,24 & 0,0055 \\
\hline Rio Grande do Norte & 93,14 & 279,99 & 0,0045 \\
\hline Rondônia & 110,54 & 340,81 & 0,0011 \\
\hline Roraima & 268,87 & 894,76 & 0,0032 \\
\hline Rio Grande do Sul & 93,85 & 255,55 & 0,0079 \\
\hline Santa Catarina & 99,18 & 277,53 & 0,0066 \\
\hline Sergipe & 96,11 & 276,97 & 0,0063 \\
\hline São Paulo & 154,27 & 465,22 & 0,0095 \\
\hline Tocantins & 136,63 & 401,68 & 0 \\
\hline BRASIL & 115,5637 & 894,76 & 0 \\
\hline Font Elata &
\end{tabular}

Fonte: Elaborada a partir de dados do Ministério da Fazenda atualizados pelo Ipeadata.

Já Minas Gerais tem gasto anual médio em torno de R \$ 95,39 per capita, o segundo menor da região Sudeste, à frente apenas do Espírito Santo ( $\mathrm{R}$ 87,68). São Paulo e Rio de Janeiro obtiveram gastos de R \$ 154,27 e R \$ 126,37, respectivamente. Os menores valores de gastos per capita na área de educação e cultura pertencem aos estados de Pernambuco e Maranhão, os quais gastaram, respectivamente, $\mathrm{R} \$ 46,17$ e $\mathrm{R} \$ 55,44$ em média, no período analisado. Ao comparar o valor per capita do Produto Interno Bruto de Roraima e Amapá (Tabela 2) aos demais estados da região Norte e Nordeste, evidenciam-se valores razoavelmente elevados. Como se observa na Tabela 3, Roraima e Amapá detêm boas médias de anos estudados, o que dá indícios de que estes tipos de gastos estão sendo canalizados adequadamente para atender as populações de ambos os estados.

Entretanto, a região Nordeste, além de não apresentar gastos públicos elevados nessa área, também não viabilizou a elevação dos mesmos, ao longo dos anos, o que induz a alguns 
Revista Economia e Desenvolvimento, v. 25, n. 2, 2013.

questionamentos. Qual tem sido a prioridade dada à educação nestes estados? Os recursos estariam sendo escassos ou mal alocados? Há outras áreas de gasto público cuja urgência de investimento é relativamente maior?

Outra variável importante ligada ao capital humano são as condições de saúde da população. Nesse sentido, a Tabela 5 informa a média da taxa de mortalidade por 1.000 nascidos, nos estados brasileiros, entre os anos de 1985 e 2008.

Tabela 5 - Taxa de mortalidade infantil por mil nascidos vivos, estados brasileiros, 1985 2008

\begin{tabular}{|c|c|c|c|}
\hline Estado & Média & Máximo & Mínimo \\
\hline Rondônia & 35,07 & 61,19 & 19,91 \\
\hline Acre & 44,12 & 71,25 & 23,39 \\
\hline Amazonas & 35,43 & 58,10 & 21,52 \\
\hline Roraima & 34,89 & 52,33 & 16,64 \\
\hline Pará & 38,66 & 65,93 & 23,09 \\
\hline Amapá & 32,61 & 49,51 & 20,85 \\
\hline Tocantins & 36,55 & 67,16 & 21,42 \\
\hline Maranhão & 58,62 & 96,27 & 24,03 \\
\hline Piauí & 50,08 & 80,87 & 23,62 \\
\hline Ceara & 54,08 & 93,44 & 19,12 \\
\hline Rio Grande do Norte & 58,89 & 103,58 & 21,08 \\
\hline Paraíba & 63,59 & 111,97 & 21,18 \\
\hline Pernambuco & 60,72 & 103,54 & 19,31 \\
\hline Alagoas & 76,23 & 166,88 & 21,53 \\
\hline Sergipe & 54,26 & 92,03 & 20,40 \\
\hline Bahia & 50,13 & 83,86 & 23,30 \\
\hline Minas Gerais & 31,04 & 58,69 & 17,38 \\
\hline Espírito Santo & 27,23 & 43,17 & 13,90 \\
\hline Rio de Janeiro & 26,32 & 44,32 & 14,31 \\
\hline São Paulo & 24,28 & 39,69 & 12,59 \\
\hline Paraná & 27,76 & 47,60 & 13,09 \\
\hline Santa Catarina & 22,84 & 38,44 & 11,69 \\
\hline Rio Grande do Sul & 19,78 & 29,25 & 12,75 \\
\hline Mato Grosso do Sul & 27,69 & 45,17 & 16,54 \\
\hline Mato Grosso & 30,35 & 50,75 & 18,23 \\
\hline Goiás & 27,69 & 46,63 & 16,89 \\
\hline Distrito Federal & 24,27 & 42,45 & 11,09 \\
\hline BRASIL & 37,75 & 66,59 & 17,60 \\
\hline
\end{tabular}

Fonte: Elaborada pelos autores com dados do Ministério da Saúde e do Instituto Brasileiro de Geografia e Estatística (IBGE), 2010.

O primeiro destaque diz respeito à região Nordeste, cujos estados detém as maiores taxas de mortalidade: Alagoas, com média de 76,23 óbitos a cada 1.000 nascidos por ano, seguido da Paraíba, Pernambuco, Rio Grande do Norte, com valores de 63,59; 60,72 e 58,89, 
Evolução dos gastos públicos estaduais em capital humano e em infraestrutura física nos Estados brasileiros.

respectivamente. A média nacional é de 37,75 , um número bem abaixo do observado no Nordeste.

Nos estados do Norte, o Acre apresenta taxa média de 44,12 e o Pará de 38,66 índices também elevados. Os estados sulistas, em geral, possuem médias baixas, a exemplo do Rio Grande do Sul e de Santa Catarina, que apresentam os menores valores do Brasil: 19,78 e 22,84, respectivamente. Analisando a região Sudeste, São Paulo detém o menor valor médio $(24,28)$, sendo que a maior taxa de mortalidade média, no período, refere-se a Minas Gerais (31,07). Observa-se que os valores máximos e mínimos para taxa de mortalidade obtiveram elevada amplitude. Alagoas desponta com o máximo de 166,88, e mínimo de 21,53 por mil nascidos vivos, o que traduz a grande melhora nesta variável ligada ao capital social. Já na Tabela 6 são apresentados os gastos estaduais em saúde e saneamento.

Tabela 6 - Gastos estaduais com saúde e saneamento, estados brasileiros, 1985-2008

\begin{tabular}{l|c|c|c}
\hline \multicolumn{1}{c|}{ Estado } & Média (R\$ milhões) & Máximo (R\$ milhões) & Mínimo (R\$) \\
\hline Acre & $111.333,05$ & $430.746,33$ & 19,7 \\
\hline Alagoas & $165.091,71$ & $701.769,40$ & 58,58 \\
\hline Amazonas & $393.553,33$ & $1.577 .784,66$ & 58,38 \\
\hline Amapá & $832.413,37$ & $355,283,59$ & 25,75 \\
\hline Bahia & $883.155,79$ & $3.213 .101,96$ & 664,74 \\
\hline Ceará & $360.404,44$ & $166.933,77$ & 42,03 \\
\hline Distrito Federal & $606.044,86$ & $1.712 .782,64$ & 259,26 \\
\hline Espírito Santo & 277.544 & $1.042 .656,77$ & 40,55 \\
\hline Goiás & $323.685,39$ & $1.401 .580,42$ & 54,63 \\
\hline Maranhão & $169.876,80$ & $974.462,32$ & 21,28 \\
\hline Minas Gerais & 923.964 & $363.476,79$ & 268,77 \\
\hline Mato Grosso do Sul & $119.788,80$ & $613.179,49$ & 37,44 \\
\hline Mato Grosso & $175.575,74$ & $791.715,99$ & 36,27 \\
\hline Pará & $362.410,24$ & $1.457 .105,33$ & 61,9 \\
\hline Paraíba & $170.021,28$ & $723.797,34$ & 15,89 \\
\hline Pernambuco & $489.994,80$ & $253.858,43$ & 133,16 \\
\hline Piaú́ & $155.473,55$ & $1.021 .946,59$ & 39,67 \\
\hline Paraná & $520.039,34$ & $2.134 .853,85$ & 121,34 \\
\hline Rio de Janeiro & $140.225,80$ & $5.220 .620,59$ & 211,01 \\
\hline Rio Grande do Norte & $217.760,50$ & $890.326,88$ & 37,95 \\
\hline Rondônia & $107.517,65$ & $443.316,46$ & 65,82 \\
\hline Roraima & $61.409,78$ & $309.691,56$ & 10,26 \\
\hline Rio Grande do Sul & $546.933,67$ & $2.014 .346,12$ & 100,39 \\
\hline Santa Catarina & $352.235,64$ & $1.223 .144,95$ & 92,64 \\
\hline Sergipe & $163.221,15$ & $627.968,44$ & $1.029,65$ \\
\hline São Paulo & $3.364 .982,64$ & $12.685 .933,34$ & $21.157,70$ \\
\hline Tocantins & $1.556 .519,59$ & $573.292,56$ & \\
\hline Brasil & $467.805,26$ & $1.851 .262,87$ & \\
\hline Fone: Ena & & \\
\hline
\end{tabular}

Fonte: Elaboração própria com base nos dados do Ipeadata (2012). 
Como observado, os estados que mais direcionam recursos para esta área são respectivamente, São Paulo, Rio de Janeiro e Minas Gerais. Este resultado pode ser, em parte, explicado pelo grande número de habitantes desses estados, o que justifica o valor absoluto relativamente maior que os demais. Outra explicação para esse fato é que tais estados representam o eixo industrial-comercial do país, permitindo-lhes auferir maior arrecadação de impostos e, por consequência, maior volume de recursos para investir na categoria analisada.

Os estados do Amazonas, Bahia e Ceará apresentam gastos públicos estaduais em saúde e saneamento superiores aos apresentados pelos estados das regiões em que se encontram, revelando, portanto, tendência diferente dos estados das regiões Norte e Nordeste de apresentarem volume de gastos inferiores. Cabe destacar que, apesar deste maior volume de gastos, não foram identificadas menores taxas de mortalidade. Nota-se também que os estados do Rio Grande do Sul e de Santa Catarina, apesar de não terem nível de gastos do Governo estadual em saúde e saneamento elevado, denotam a menor taxa de mortalidade infantil. Este fato pode indicar maior eficácia na alocação dos recursos nestas federações. Ademais, os montantes mínimos estaduais são valores muito abaixo das médias, o que demonstra que houve períodos de investimento praticamente nulo na área.

A próxima análise tem como base a Tabela 7, que trata de dados relativos à infraestrutura física do país, especificamente, da pavimentação das estradas em cada estado. Para isso, foi calculada a média dos quilômetros pavimentados de rodovias federais, entre os anos de 1985 a 2008. 
Evolução dos gastos públicos estaduais em capital humano e em infraestrutura física nos Estados brasileiros.

Tabela 7 - Quilômetros pavimentados de rodovias federais nos estados brasileiros, 1985-2008

\begin{tabular}{|c|c|c|c|}
\hline Estado & Média & Máximo & Mínimo \\
\hline Rondônia & 1.119 & 1.363 & 713 \\
\hline Acre & 420 & 1.157 & 194 \\
\hline Amazonas & 621 & 857 & 262 \\
\hline Roraima & 504 & 1.048 & 40 \\
\hline Pará & 1.083 & 1.723 & 637 \\
\hline Amapá & 190 & 342 & 82 \\
\hline Tocantins & 1.018 & 1.458 & 752 \\
\hline Maranhão & 2.611 & 3.683 & 1.807 \\
\hline Piauí & 2.202 & 2.464 & 1.978 \\
\hline Ceará & 2.162 & 2.747 & 1.788 \\
\hline Rio. Grande do Norte & 1.321 & 1.537 & 1.073 \\
\hline Paraíba & 1.274 & 1.461 & 1.064 \\
\hline Pernambuco & 2.477 & 2.523 & 2.353 \\
\hline Alagoas & 730 & 757 & 681 \\
\hline Sergipe & 318 & 323 & 312 \\
\hline Bahia & 4.502 & 5.431 & 3.717 \\
\hline Minas Gerais & 9.991 & 12.376 & 8.286 \\
\hline Espírito Santo & 884 & 1.060 & 713 \\
\hline Rio de Janeiro & 1.687 & 2.011 & 1.509 \\
\hline São Paulo & 2.280 & 5.361 & 1.085 \\
\hline Paraná & 3.470 & 4.491 & 2.837 \\
\hline Santa Catarina & 2.231 & 2.795 & 1.899 \\
\hline Rio Grande do Sul & 5.307 & 6.903 & 4.907 \\
\hline Mato Grosso do Sul & 2.974 & 3.751 & 2.253 \\
\hline Mato Grosso & 2.828 & 3.501 & 1.976 \\
\hline Goiás & 3.297 & 4.418 & 2.610 \\
\hline Distrito Federal & 232 & 317 & 161 \\
\hline Brasil & 58.891 & 74.070 & 46.455 \\
\hline
\end{tabular}

Fonte: Elaborada pelos autores com dados do IBGE e do Departamento Nacional de Infraestrutura de Transportes (DNIT), 2012.

Primeiramente, percebe-se que Minas Gerais é o estado com melhor média anual de estradas pavimentadas: $9.981 \mathrm{~km}$. Em segundo lugar, aparece o Rio Grande do Sul, com $5.307 \mathrm{~km}$ de estradas federais pavimentadas, sendo que a Bahia e o Paraná alcançam médias de 4.502 e 3.470 quilômetros, respectivamente. Sob este aspecto, o pior estado é o Piauí, com apenas $190 \mathrm{~km}$, seguido do Distrito Federal $(232 \mathrm{~km})$ e de Sergipe, com $318 \mathrm{~km}$. A explicação para o caso do Distrito Federal é sua pequena área territorial. A média nacional é de $58.891 \mathrm{~km}$, tendo sido identificados dois outliers, um positivo (Minas Gerais) e outro negativo (Piauí). Em geral, evidencia-se que a malha rodoviária nacional é extensa, no entanto, a avaliação da qualidade das pavimentações não pode ser feita, a partir deste tipo de parâmetro. Como complementos à Tabela 7, são apresentados na Tabela 8 os dados sobre gastos com transporte rodoviário, para cada estado brasileiro, entre 1995 e 2008. 
Revista Economia e Desenvolvimento, v. 25, n. 2, 2013.

Tabela 8 - Gastos públicos com o transporte rodoviário (R\$ mil), estados do Brasil, 1995 2008

\begin{tabular}{l|c|c|c}
\hline \multicolumn{1}{c|}{ Estado } & Média & Máximo & Mínimo \\
\hline Acre & 62.459 & 199.959 & 15.654 \\
\hline Amapá & 25.184 & 84.804 & 0 \\
\hline Amazonas & 35.661 & 149.655 & 3.865 \\
\hline Pará & 78.076 & 153.576 & 13.585 \\
\hline Rondônia & 32.958 & 68.721 & 8.950 \\
\hline Roraima & 36.332 & 166.351 & 2.991 \\
\hline Tocantins & 50.503 & 137.851 & 5.613 \\
\hline Alagoas & 18.731 & 81.196 & 7.303 \\
\hline Bahia & 99.312 & 247.285 & 24.880 \\
\hline Ceará & 46.336 & 114.794 & 9.326 \\
\hline Maranhão & 50.038 & 112.848 & 16.249 \\
\hline Paraíba & 53.157 & 274.498 & 2.862 \\
\hline Pernambuco & 87.328 & 363.901 & 8.181 \\
\hline Piauí & 28.998 & 64.025 & 1.560 \\
\hline Rio Grande do Norte & 40.133 & 137.905 & 4.679 \\
\hline Sergipe & 13.023 & 49.534 & 907 \\
\hline Espírito Santo & 32.738 & 74.715 & 11.305 \\
\hline Minas Gerais & 405.357 & 789.995 & 52.587 \\
\hline Rio de Janeiro & 37.616 & 171.673 & 9.080 \\
\hline São Paulo & 158.976 & 333.611 & 23.596 \\
\hline Paraná & 91.047 & 158.468 & 29.400 \\
\hline Rio Grande do Sul & 140.546 & 364.009 & 44.952 \\
\hline Santa Catarina & 178.393 & 506.909 & 25.867 \\
\hline Distrito Federal & 12.562 & 54.785 & 670 \\
\hline Goiás & 120.089 & 288.933 & 29.137 \\
\hline Mato Grosso & 88.194 & 227.444 & 21.198 \\
\hline Mato Grosso do Sul & 82.947 & 273.803 & - \\
\hline BRASIL & 78.026 & - & \\
\hline Font Erabara & & \\
\hline
\end{tabular}

Fonte: Elaborada pelos autores com dados do Ministério dos Transportes.

Minas Gerais destaca-se em primeiro lugar como o estado que mais despende recursos no setor rodoviário, $\mathrm{R} \$ 405.357$ milhões anuais, consonante à vasta rede de rodovias pavimentadas. Em segundo lugar está Santa Catarina, com R\$178.393 milhões e, em terceiro, São Paulo, com R\$ 158.976 milhões. O Distrito Federal (DF) apresenta o menor valor de despesas nesta categoria: $\mathrm{R} \$ 12.562$ milhões. Isso explica o fato de o DF possuir a segunda menor extensão de rodovias pavimentas do país, perdendo apenas para o Amapá. As regiões Norte e Nordeste caracterizam-se por apresentarem baixos valores de gasto público nesta categoria. Um exemplo é o Sergipe, cujo montante é de R \$ 13.023 milhões. Grande parte das unidades federativas dessas duas regiões ficou com gastos abaixo da média nacional, que foi de R $\$ 78$ milhões. 
Evolução dos gastos públicos estaduais em capital humano e em infraestrutura física nos Estados brasileiros.

Deve-se destacar que os estados que compõem a Floresta Amazônica apresentam valores de dispêndios extremamente baixos em transporte rodoviário, a exemplo do Amazonas (R\$35.661 milhões). Tal evidência pode ser, em parte, explicada pelo fato dessa região deter extensão de rios muito elevada e, por conseguinte, ter como principal meio de transporte o tipo fluvial. Entretanto, esse não é o único fator responsável. O baixo investimento em transporte público por parte do Estado também é uma justificativa desse resultado. Pode ser evidenciado na infraestrutura das hidrovias brasileiras. Tal observação justifica a dificuldade de comparação de extensão rodoviária com outras regiões, já que o meio de transporte é distinto do usado na maioria do país: o transporte rodoviário. A seguir, na Tabela 9, é possível observar o elenco dos gastos públicos estaduais em energia de 1985 a 2008.

Tabela 9 - Gastos públicos estaduais com energia, estados brasileiros, 1985-2008

\begin{tabular}{|c|c|c|c|}
\hline Estado & Média (Milhões R\$) & Máximo (milhões R\$) & Mínimo (R\$) \\
\hline Acre & 664,65 & $3.481,17$ & 0 \\
\hline Alagoas & $1.937,80$ & $34.858,62$ & 0 \\
\hline Amazonas & $5.564,08$ & $51.119,67$ & 0 \\
\hline Amapá & $2.675,65$ & $15.986,61$ & 0 \\
\hline Bahia & $29.041,16$ & $114.656,26$ & 84.042 \\
\hline Ceará & $15.468,89$ & $127.739,22$ & 3.145 \\
\hline Distrito Federal & $15.870,12$ & $96.242,65$ & 0 \\
\hline Espírito Santo & 135,51 & 2.544 & 0 \\
\hline Goiás & $1.116,12$ & $10.252,04$ & 15.030 \\
\hline Maranhão & $7.398,90$ & $96.299,02$ & 0 \\
\hline Minas Gerais & $4.644,90$ & 62.692 & 0 \\
\hline Mato Grosso do Sul & 756,96 & $12.466,52$ & 0 \\
\hline Mato Grosso & $2.682,18$ & $14.523,37$ & 0 \\
\hline Pará & $6.317,58$ & $58.421,75$ & 0 \\
\hline Paraíba & $2.961,82$ & $17.611,59$ & 11.3220 \\
\hline Pernambuco & 619,93 & 7.958 & 18.755 \\
\hline Piauí & 446,31 & $6.677,74$ & 0 \\
\hline Paraná & 747,35 & $3.219,71$ & 0 \\
\hline Rio de Janeiro & $3.010,25$ & $25.224,42$ & 0 \\
\hline Rio Grande do Norte & $9.268,48$ & $88.389,27$ & 6.519 \\
\hline Rondônia & 354,29 & $4.293,80$ & 0 \\
\hline Roraima & $14.415,8$ & $94.682 .723,7$ & 0 \\
\hline Rio Grande do Sul & $3.722 .362,2$ & 19.122 .281 & 36.850 \\
\hline Santa Catarina & $680.249,12$ & $15.001 .731,1$ & 0 \\
\hline Sergipe & $754.944,95$ & $10.325 .423,7$ & 0 \\
\hline São Paulo & 184.397 .113 & 1.494 .553 .514 & 114.161 \\
\hline Tocantins & $9.779 .237,7$ & $83.834,42$ & 0 \\
\hline BRASIL & $12.053,06$ & $60.541 .635,75$ & 10.734 \\
\hline
\end{tabular}

Fonte: Elaboração própria com base nos dados do Ipeadata (2012). 
Percebe-se que o estado de São Paulo apresenta média de gastos públicos estaduais em energia bem superior aos demais estados, devido, em parte, ao grande parque industrial neste estado presente. Notam-se ainda as diferenças entre os gastos dos outros estados, destacandose a Bahia, como o segundo estado que mais direciona recursos para energia. Apesar disso, o estado baiano ainda fica muito aquém de São Paulo. Assim como os gastos públicos em saúde e saneamento, porém com maior intensidade, os gastos em energia apresentam valores mínimos muito baixos. São valores nulos em diversos, em algum ano da análise, e nos estados que não apresentam valores nulos, os valores mínimos chegam próximos de zero. Isso indica que a falta de investimentos em energia explica parcela significativa do racionamento no começo do século XXI, realçando a fragilidade da infraestrutura brasileira, prejudicando, portanto, a produtividade do setor privado e a geração de emprego e renda.

Observando-se os dados dos investimentos em setores da infraestrutura brasileira, pode-se identificar períodos muito longos de falta de recursos, além da grande disparidade interestadual. Este tipo de dispêndio somado aos investimentos nessa área é essencial para a geração de crescimento econômico, sendo importantes também para melhora da qualidade de vida da população. No que diz respeito às condições de sobrevivência da população brasileira, cabe avaliar as Tabelas 10 e 11, que trazem dados sobre o número de pessoas vivendo na extrema pobreza e o número de pobres, respectivamente, entre 1985 e 2008. 
Evolução dos gastos públicos estaduais em capital humano e em infraestrutura física nos Estados brasileiros.

Tabela 10 - Número de pessoas extremamente pobres nos estados brasileiros, 1985-2008

\begin{tabular}{l|c|c|c}
\hline \multicolumn{1}{c|}{ Estado } & Média & Máximo & Mínimo \\
\hline Acre & 67.615 & 139.732 & 6.947 \\
\hline Alagoas & 874.705 & 1.160 .994 & 393.626 \\
\hline Amazonas & 340.358 & 604.419 & 66.704 \\
\hline Amapá & 51.099 & 114.130 & 6.588 \\
\hline Bahia & 3.512 .376 & 4.653 .413 & 1.603 .527 \\
\hline Ceará & 2.195 .369 & 2.850 .050 & 1.151 .578 \\
\hline Distrito Federal & 128.565 & 239.399 & 50.175 \\
\hline Espírito Santo & 276.988 & 508.076 & 70.539 \\
\hline Goiás & 389.702 & 618.052 & 135.744 \\
\hline Maranhão & 1.905 .356 & 2.353 .085 & 1.142 .612 \\
\hline Minas Gerais & 1.579 .583 & 2.403 .958 & 659.198 \\
\hline Mato Grosso do Sul & 140.326 & 212.639 & 30.350 \\
\hline Mato Grosso & 185.328 & 302.587 & 43.362 \\
\hline Pará & 652.149 & 1.148 .570 & 152.738 \\
\hline Paraíba & 1.046 .561 & 1.525 .126 & 581.627 \\
\hline Pernambuco & 2.146 .829 & 2.701 .373 & 1.096 .841 \\
\hline Piauí & 1.078 .603 & 1.513 .511 & 569.129 \\
\hline Paraná & 953.101 & 1.510 .054 & 385.362 \\
\hline Rio de Janeiro & 980.210 & 1.537 .318 & 484.633 \\
\hline Rio Grande do Norte & 694.419 & 911.374 & 398.882 \\
\hline Rondônia & 100.251 & 183.198 & 13.384 \\
\hline Roraima & 29.784 & 115.320 & 786 \\
\hline Rio Grande do Sul & 810.016 & 1.291 .421 & 387.059 \\
\hline Santa Catarina & 286.993 & 596.244 & 188.074 \\
\hline Sergipe & 399.938 & 517.864 & 542.122 \\
\hline São Paulo & 1.714 .729 & 2.737 .480 & 384,454 \\
\hline Tocantins & 262.730 & 389.252 & \\
\hline BRASIL & 844.581 & 1.216 .246 & \\
\hline Fona & & & \\
\hline
\end{tabular}

Fonte: Elaboração própria com base nos dados do Ipeadata (2012).

Nota: *Para os anos 1991, 1994 e 2000 não há dados disponíveis. Para Tocantins, não há dados de 1985 a1990.

Para 1986, não há dados para Amapá e Roraima, e para 2007, não há informações do Distrito Federal.

Segundo os dados da Tabela 10, a Bahia é o estado que possui, em média, o maior número de pessoas vivendo na extrema pobreza, durante o período considerado. Este estado também apresenta o maior valor máximo da série, apesar de demonstrar tendência de queda, ao longo do período, com valor mínimo, em 2008. As unidades federativas de Ceará, Maranhão, Minas Gerais, Piauí e Tocantins, apesar de possuírem alto número de pessoas extremamente pobres, também apresentaram valores mínimos em 2008. Isso dá indícios de relativa eficácia das políticas empregadas de combate à pobreza absoluta, a exemplo das políticas de transferência de renda e outros tipos de gastos públicos. 
Tabela 11 - Número de pessoas pobres nas unidades federativas, 1985-2008*

\begin{tabular}{|c|c|c|c|}
\hline Estado & Média & Máximo & Mínimo \\
\hline Acre & 153.916 & 313.860 & 36.025 \\
\hline Alagoas & 1.696 .451 & 2.004 .727 & 1.203 .208 \\
\hline Amazonas & 853.889 & 1.457 .689 & 221.518 \\
\hline Amapá & 127.915 & 256.153 & 4.379 \\
\hline Bahia & 7.081 .916 & 8.075 .509 & 4.793 .056 \\
\hline Ceará & 4.127 .419 & 4.558 .227 & 3.036 .211 \\
\hline Distrito Federal & 390.782 & 587.226 & 182.729 \\
\hline Espírito Santo & 778.662 & 1.102 .995 & 373.108 \\
\hline Goiás & 1.229 .082 & 1.624 .596 & 599.461 \\
\hline Maranhão & 3.425 .383 & 3.908 .741 & 2.354 .642 \\
\hline Minas Gerais & 4.609 .161 & 5.972 .603 & 2.521 .136 \\
\hline Mato Grosso do Sul & 479.053 & 628.324 & 196.417 \\
\hline Mato Grosso & 578.380 & 788.597 & 225.757 \\
\hline Pará & 1.761 .999 & 3.270 .370 & 600.398 \\
\hline Paraíba & 1.976 .351 & 2.328 .600 & 1.520 .633 \\
\hline Pernambuco & 4.306 .257 & 4.972 .574 & 3.037 .789 \\
\hline Piauí & 1.771 .288 & 2.017 .407 & 1.316 .103 \\
\hline Paraná & 2.646 .358 & 3.539 .397 & 1.396 .089 \\
\hline Rio de Janeiro & 3.210 .863 & 4.405 .416 & 1.970 .251 \\
\hline Rio Grande do Norte & 1.428 .544 & 1.647 .755 & 1.062 .247 \\
\hline Rondônia & 287.185 & 555.303 & 42.474 \\
\hline Roraima & 73.426 & 208.831 & 1.544 \\
\hline Rio Grande do Sul & 2.385 .828 & 3.296 .110 & 1.463 .551 \\
\hline Santa Catarina & 952.464 & 1.440 .298 & 449.945 \\
\hline Sergipe & 860.535 & 991.491 & 562.086 \\
\hline São Paulo & 5.928 .208 & 8.113 .404 & 2.484 .845 \\
\hline Tocantins & 576.313 & 671.911 & 393.491 \\
\hline Brasil & 1.988 .801 & 2.545 .856 & 1.187 .003 \\
\hline
\end{tabular}

Fonte: Elaboração própria com base nos dados do Ipeadata (2012).

Nota: *Para os anos 1991, 1994 e 2000 não há dados disponíveis, sendo que para o Tocantins também não há dados do período 1985-1990, e no ano de 2007 para o Distrito Federal.

É necessário ressaltar que estes dados referem-se a valores absolutos, sendo a comparação entre anos inviável devido às divergências de crescimento populacional estadual. Entretanto, considerando-se que a taxa de fecundidade brasileira sempre apresentou valores positivos, o valor mínimo absoluto no final do período considerado é significativo. Outro estado que apresenta alto valor de indivíduos extremamente pobres é São Paulo, o que pode estar atrelado à migração para esse advinda principalmente das regiões Norte e Nordeste. Esses indivíduos acabam marginalizados devido a pouca educação e qualificação profissional que possuem, vivendo em áreas precárias e de segurança comprometida, tendo dificuldades de acesso aos serviços básicos de saúde e educação, entre outros quesitos igualmente importantes. Os estados de Ceará, Maranhão, Minas Gerais, Pernambuco e Tocantins 
Evolução dos gastos públicos estaduais em capital humano e em infraestrutura física nos Estados brasileiros.

apresentam significativos valores, tanto para pessoas extremamente pobres, quanto para pessoas pobres. O estado de Minas Gerais apresenta o pior resultado entre os estados da região sudeste, tanto para índices de pobreza, quanto para valores do PIB per capita. O Piauí possui elevado número de pessoas vivendo na extrema pobreza, embora não tenha um número tão elevado de pessoas pobres, se comparado a outros estados.

\section{5 - CONSIDERAÇÕES FINAIS}

Diante dos objetivos propostos neste estudo, fica atestada, por meio da análise de estatística descritiva, a eficácia dos dispêndios públicos em infraestrutura rodoviária e em educação e saúde quanto aos avanços na malha rodoviária e no estoque de capital humano, respectivamente, a partir da análise da estrutura socioeconômica dos estados brasileiros. A análise descritiva da evolução de variáveis socioeconômicas relevantes abordadas no estudo confirma a disparidade de dinamismo econômico e de infraestrutura social entre as unidades da federação. Fica claro que os estados do Sul e Sudeste continuam a concentrar riqueza e maior infraestrutura em detrimento dos demais. Entretanto, houve melhora geral no país, nos anos de 2007 e 2008, em relação à década de 1980. Um exemplo é a diminuição da taxa de mortalidade em todos os estados. O mesmo pode-se afirmar sobre os indicadores de escolaridade média, PIB per capita, condição das rodovias federais e de concentração de renda, cujos índices indicam melhora na maioria das regiões. Todavia, apesar dos avanços, a análise aponta alguns problemas comuns aos estados e mostra certo direcionamento de quais indicadores sociais e econômicos carecem de políticas públicas mais efetivas, a exemplo da crônica pobreza das regiões Norte e Nordeste do Brasil.

É importante atentar, também, que as políticas de gasto social, a exemplo do Programa Bolsa Família, permite aos seus beneficiários a possibilidade de obter suporte financeiro atrelado ao cumprimento de condicionalidades de serviços básicos de educação e saúde. No entanto, apesar dos resultados positivos, sobretudo, de curto prazo, deste tipo de instrumento político, a renda é instrumento de obtenção de capacidades, mas não o fim. A proposta de ligar o recebimento do benefício mensal ao acesso a itens de capital social é de grande mérito, pois, permite elevar o potencial da população pobre de obter renda, ou seja, dá possibilidade aos pobres de se libertarem da pobreza medida, essencialmente, pela renda.

No entanto, as políticas de gasto social pautadas em concessão de benefícios financeiros trazem à tona outra problemática: a dificuldade de inserção efetiva da população mais pobre na economia, de modo a poderem desfrutar dos resultados positivos do processo 
de crescimento econômico. A verdade é que os retornos aos ativos, a exemplo da educação, dependem, fortemente, da provisão de ativos públicos complementares como, por exemplo, infraestrutura escolar e qualidade de ensino. No entanto, torna-se evidente que, em grande parte do Brasil, os cidadãos pobres têm acesso a ensino de baixa qualidade, e com isso, os retornos à educação são, relativamente, mais baixos para esses indivíduos. A priori, não estando preparada para o mercado de trabalho, esta parcela da população tem reduzida sua probabilidade de acumulação de capital humano e de mobilidade social, fatores essenciais para o crescimento e alívio da pobreza nacional.

Outro agravante é o fato de o século XXI apresentar-se como o tempo das inovações, da valorização do conhecimento, de modo que a constante revolução na fronteira tecnológica provoca aumento da demanda por trabalhadores mais qualificados. Deste modo, retirar a parcela da população da condição de pobreza estritamente ligada à renda não soluciona o entrave relacionado à possibilidade de aumento dos rendimentos dos trabalhadores mais qualificados para a inovação tecnológica, o que aumenta o hiato de salários entre estes e os não qualificados (grande parte, indivíduos pobres). Logo, a parcela de indivíduos que sai do status de "pobre" atrelado ao conceito de linha de pobreza não se integra, efetivamente, ao mercado de trabalho e à dinâmica da sociedade de consumo, o que acaba por agravar a desigualdade social no Brasil. Surgem então para os formuladores de políticas obstáculos adicionais relacionados ao desafio de atenuar a distorção gerada por esse tipo de instrumento de crescimento. Em suma, realça-se a importância da atuação do Estado, em outras instâncias, por meio de outros tipos de políticas públicas que permitam implantar, efetivamente, o crescimento do tipo pró-pobre, que não apenas os instrumentos de apoio financeiro.

\section{6- REFERÊNCIAS}

ASCHAUER, D. Is public expenditure productive? Journal of Monetary Economics, v. 23, p. 177-200, 1989.

BARRO, R. J. Government spending in a simple model of endogenous growth. Journal of Political Economy, v. 98, p. 103-125, 1990.

BARROS, R. P.; HENRIQUES, R.; MENDONÇA, R. A Estabilidade Inaceitável:

Desigualdade e Pobreza no Brasil. Rio de Janeiro: IPEA, 2001. (Texto para discussão IPEA, no. 800).

BARROS, R; HENRIQUES, R; MENDONÇA, R. A Estabilidade Inaceitável: desigualdade e pobreza no Brasil. In: Henriques, Ricardo (Org.). Desigualdade e Pobreza no Brasil. cap. 1, pp. 21-47. IPEA: Rio de Janeiro, 2000a. 
Evolução dos gastos públicos estaduais em capital humano e em infraestrutura física nos Estados brasileiros.

BARROS, R; HENRIQUES, R; MENDONÇA, R. A (2000b). Pobreza e política social. São Paulo, Fundação Konrad Adenauer, 2000b.

BARROS, R. P. \& MENDONÇA, R. O impacto do crescimento e de reduções no grau de desigualdade sobre a pobreza. Rio de Janeiro: IPEA, 1997. (Texto para Discussão 528)

BLACKBURN, K.; HUNG, V. T. Y. E POZZOLO, A. F. Research, Development and Human Capital Accumulation" - Journal of Macroeconomics, volume 22, number 2, 2000.

BOURGUIGNON, F. Pareto-Superiority of Unegalitarian Equilibrium in Stiglitz'Model of Wealth Distribution with Convex Savings Function, Econometrica 49, 1981.

BOURGUIGNON, F. The Growth Elasticity of Poverty Reduction; Explaining Heterogeneity Across Countries and Time Periods in T. Eicher and S. Turnovsky, eds. Inequality and growth. Theory and Policy Implications, Cambridge: The MIT Press, 2003.

CASTRO, J. A. Evolução e desigualdade na educação brasileira. Educação e Sociedade, Campinas, vol. 30, n. 108, 2009.

CRUZ, A. C.; TEIXEIRA, E. C.; BRAGA, M. J. O efeito dos gastos públicos em infraestrutura e em capital humano no crescimento econômico e na redução da pobreza no Brasil. Economia (Brasília), v. 11, p. 163-185, 2010.

FERRANTI, D. et al. Inequality in Latin America: breaking with history? Washington, DC: World Bank, 2004.

FERREIRA, A. B.; NAKABASHI, L.; SANTOS, M. Crescimento econômico e acumulação de capital humano: uma análise de causalidade. Belo Horizonte: UFMG/CEDEPLAR, 2003. (Texto para discussão n. 222)

FERREIRA, F. H. G. Os determinantes da desigualdade de renda no Brasil: luta de classes ou heterogeneidade educacional? Rio de Janeiro, Departamento de Economia PUC, Texto para discussão n. $^{\circ} 415,2000$.

FERREIRA, P.C. Investimento em infraestrutura no Brasil: fatos estilizados e relações de longo prazo. Pesquisa e Planejamento Econômico, v. 26, no. 2, 231-252, 1996.

FERREIRA, P.C.; MALLIAGROS, T.G. Impactos produtivos da infraestrutura no Brasil 1950/95. Pesquisa e Planejamento Econômico, v.28 n.2, 315-338, 1998.

FOSTER, A.D., ROSENZWEIG, M.R. Technical Change and Human-Capital Returns and Investments: Evidence from the Green Revolution. The American Economic Review, 86 (4), p. 931-953, 1996.

GALOR, O.; ZEIRA, J. Income Distribution and Macroeconomics. Review of Economic Studies, 60, p. 35-52, 1993.

HIRSCHMAN, A. O. Estratégia do Desenvolvimento Econômico. Rio de Janeiro, Fundo de Cultura, 1961.

HOFFMANN, R. Elasticidade da pobreza em relação à renda média e à desigualdade no Brasil e nas unidades da Federação. Economia Revista da Anpec, v. 6, n. 2, p. 255-289, 2005. 
IBGE (2012) Instituto Brasileiro de Geografia e Estatística:

http://www.ibge.gov.br/home/php. Acesso em novembro de 2012.

IPEADATA (2012). Disponível em: http://www.ipeadata.gov.br/. Acesso em abril de 2012.

KAKWANI, N.; PERNIA, E. What is Pro-Poor Growth?. Asian Development Review 16(1), 2000.

KALDOR, N. Alternative Theories of Distribution, Review of Economic Studies, 23, 1956.

KELLER, W. International Technology Diffusion. Journal of Economic Literature. Under revision, 2003.

LOAYZA, N., FAJNZYLBER, P., CALDERÓN, C. "Economic Growth in Latin America and The Caribbean: Stylized Facts, Explanations, and Forecasts, "Working Papers Central Bank of Chile 265, Central Bank of Chile, 2004

LIMA, F. S.; BARRETO, F. A.; MARINHO, E. Impacto do Crescimento Econômico e da Concentração de Renda sobre o Nível de Pobreza dos Estados Brasileiros. Anais do VII Encontro Regional de Economia, Fortaleza. 2003.

LOPEZ, H. Pro-poor-Pro-growth: Is there a Trade Off?. The World Bank, Policy Research Working, Paper No. 3378, 2004a.

LOPEZ, H.; SERVEN, L. The Mechanics of Growth-Poverty-Inequality Relationship. Mimeo, The World Bank, 2004b.

LOPEZ, J. H. Pro-poor growth: A review of what we know (and of what we don't). PREM Poverty Group Paper. Washington DC: The World Bank, 2004b.

MANSO, C. A.; BARRETO, F. A.O Desequilíbrio Regional de Bem-Estar Social no Brasil: Implicações a Partir do Mercado de Trabalho Durante os Ciclos Econômicos. Ensaio Sobre Pobreza, N. ${ }^{19}$, 2009.

MENEZES, T. A.; PINTO, R. F. É Preciso Esperar o Bolo Crescer, para Depois Repartir? Anais do VIII Encontro Regional de Economia, Fortaleza, 2005.

MINCER, J. Investment in Human Capital and Personal Income Distribution. Journal of Political Economy, 66(4), p. 281-302, 1958.

MIRRLEES, J. An Exploration in the Theory of Optimum Income Taxation. The Review of Economic Studies, Vol. 38, No. 2, pp. 175-208, 1971.

RAM, R. Government size and economic growth: a new framework and some evidence from cross-section and time-series data. American Economic Review, v. 76, n. 1, p. 191-203, 1986.

RAVALLION, M.; CHEN, S. Measuring Pro-poor Growth, Economic Letters , Vol. 78 (1), 2003.

RAY, D. Development Economics. Princeton, NJ: Princeton University Press, 1998. 
Evolução dos gastos públicos estaduais em capital humano e em infraestrutura física nos Estados brasileiros.

Rocha S 2011. O programa Bolsa Família Evolução e efeitos sobre a pobreza, Economia e Sociedade, Campinas, v. 20, n. 1 (41), p. 113-139, abr. 2011.

SEN, A. (1983). Development: Which Way Now? The Economic Journal 93(372): 745-762.

SILVA, G. J. C.; FORTUNATO, W. L. L. Infraestrutura e crescimento: uma avaliação do caso brasileiro no período 1985-1998. UFMG, 2002.

VANDENBUSSCHE, J., AGHION, P.; MEGHIR, C. Growth, distance to frontier and composition of human capital. Journal of Economic Growth 11, no. 2, p. 97-127 2006.

WHITE, H.; ANDERSON, A. Growth vs. Redistribution: Does the Pattern of Growth Matter?. DFID white paper on Elimination World Poverty; making Globalization Work for the Poor, 2000. 